\title{
UNA LECTURA CRÍTICA SOBRE EL CONCEPTO DE "EXTRACTIVISMO" EN EL MARCO DE LOS PROCESOS DE ACUMULACIÓN
}

Revista Trama

Volumen 7, número 2

Julio - Diciembre 2018

Páginas 108-117

ISNN-1659-343X

http://revistas.tec.ac.cr/trama
A Critical Reading On The Concept Of "Extractivism" Within The Framework Of The Accumulation Processes

Guido Pascual Galafassi ${ }^{1}$

Lorena Natalia Riffo ${ }^{2}$

Fecha de recepción: 10 de abril de 2018 Fecha de aprobación: 18 de setiembre de 2018

Galafassi, G. y Riffo, N. (2018). Una lectura crítica sobre el concepto de "extractivismo" en el marco de los procesos de acumulación, Trama, revista de ciencias sociales y humanidades, Volumen 7, (2), págs. 108-117.

DOI: https://doi.org/10.18845/tramarcsh.v7i2.3939
1. Profesor-investigador. Grupo de Estudios sobre Acumulación, Conflictos y Hegemonía - Universidad Nacional de Quilmes y Consejo Nacional de Investigaciones Científicas y Técnicas de Argentina. Quilmes, Buenos Aires, Argentina. Correo electrónico: ggalafassi@unq.edu.ar

2. Profesora-Investigadora. Instituto Patagónico de Estudios en Humanidades y Ciencias Sociales-Universidad Nacional del Comahue, Neuquen Capital, Neuquen, Argentina. - Consejo Nacional de Investigaciones Científicas y Técnicas de Argentina, Facultad de Derecho y Ciencias Sociales-Universidad Nacional del Comahue y Grupo de Estudios sobre Acumulación, Conflictos y Hegemonía Universidad Nacional de Quilmes. Quilmes, Buenos Aires, Argentina. Correo electrónico: lorenanriffo@gmail.com 
Resumen

Para percibir y entender toda la complejidad del proceso de relación sociedad-naturaleza-territorio es necesario tomar el proceso extractivo, en tanto integrante de un complejo entramado de relaciones, operaciones y procesos que adoptan las formaciones sociales en tanto estrategia de producción, distribución y reproducción de los recursos (naturales y humanos), los beneficios y el trabajo. Por esto, resulta indispensable pensar al proceso extractivo (en tanto práctica), más que al extractivismo (en tanto fenómeno sustantivo) como una etapa del proceso total de la acumulación. Y como etapa, va sufriendo -al igual que el proceso de acumulación- cambios y transformaciones a lo largo del tiempo, pero siempre en relación con los principios básicos que implican tanto la explotación del trabajo como de la naturaleza.

Palabras Clave: extractivismo, acumulación, sociedad, naturaleza.

\section{Abstract}

To perceive and understand all the complexity of the process of society-nature-territory relationship, it is necessary to take the extractive process as a component of a complex network of relationships. This process is based on operations and processes adopted by the social formations as a strategy of production, distribution and reproduction of resources (natural and human), benefits and work. For this reason, it is essential to think of the extractive process (as a practice), rather than extractivism (as a substantive phenomenon) as a stage in the total process of accumulation. And as a stage, it suffers -as well as the process of accumulationchanges and transformations over time, but always in relation to the basic principles that imply both the exploitation of work and nature.

Keywords: extractivism, accumulation, society, nature. 
I. INTRODUCCIÓN: EL "EXTRACTIVISMO" COMO EXPLICACIÓN DE LA CRISIS AMBIENTAL DEL PRESENTE

Para percibir y entender toda la complejidad del proceso de relación sociedad-naturaleza-territorio es necesario tomar el proceso extractivo, como integrante de un complejo entramado de relaciones, operaciones y procesos que adoptan las formaciones sociales en tanto estrategia de producción, distribución y reproducción de los recursos (naturales y humanos), los beneficios y el trabajo (Marx, ([1867] 1998). Por esto, resulta indispensable pensar al proceso extractivo (en tanto práctica), más que al extractivismo (en tanto fenómeno sustantivo) como una etapa del proceso total de la acumulación. Y como etapa, va sufriendo -al igual que el proceso de acumulación- cambios y transformaciones a lo largo del tiempo, pero siempre en relación con los principios básicos que implican tanto la explotación del trabajo como de la naturaleza (1 ra y 2 da contradicción del capital).

Si el proceso de acumulación capitalista tiene ya casi cinco siglos de existencia es obvio esperar que el proceso extractivo se haya modificado ampliamente, atendiendo especialmente al dinámico ritmo de innovación tecnológica que caracteriza al capital y a los cambios culturales asociados. Sin embargo, esto de ninguna manera implica que con cada renovación se acceda a un nuevo (neo) proceso extractivo ni nuevo (neo) proceso de acumulación. ${ }^{3}$ La lógica capitalista que subyace no deja de asentarse en tanto estrategia de explotación y dominación, en la extracción de plusvalía y producción de desigualdad al separar medios de producción y fuerza de trabajo manteniendo una construcción de sentido en términos de pensar la igualdad solo en el plano normativo.

La articulación sociedad-naturaleza-territorio debe entenderse como mediación dialéctica. Es la mediación social la forma de articulación existente entre los mundos físico-biológicos y el mundo humano histórico-cultural (que incluye dialécticamente al primero), y es irremediablemente mediación pues cada uno de ellos, si bien conforman la unidad diversa naturaleza-cultura/historia, se configura con base en premisas particulares y características singulares. Esta articulación sociedad-naturaleza-territorio y esta unidad dialéctica de la existencia implica siempre el aprovechamiento de la naturaleza por la sociedad más sus diversas formas de representarla y la consecuente construcción social de un territorio.

El ser humano en sociedad tiene desde siempre la capacidad de "trascender histórico-culturalmente" las leyes ecosistémicas, convirtiéndose así en sujeto que interactúa con la materia y el espacio, los piensa y los transforma (Marx, [1844] 1968, [1857] 1971). Esta transformación implica la valorización y utilización de esta materia, la representación y extracción de componentes de la naturaleza y los resignifica al introducirlos en su propio proceso de producción y reproducción en relación siempre a un régimen de acumulación predominante (material y simbólico); procesos que contienen al mismo tiempo la construcción de uno y múltiples territorios. Esta transformación permanente y creciente, implica necesariamente un proceso social, histórico y cultural de construcción del territorio a partir de un espacio dado naturalmente o ya previamente transformado, un territorio así, que se hace moldeando y remodelando el espacio natural en pos de su aprovechamiento.

Esta construcción y reconstrucción territorial se hace siempre sobre la base de la extracción de recursos de la naturaleza, extracción que es inherente al ser de lo humano sobre la tierra, pero que se enhebra en cada momento histórico y en cada espacio con determinados patrones de acumulación que son aquellos que definirán tipo e intensidad de esta extracción. Así, el proceso de extracción nunca es la variable independiente del proceso. Y esta construcción está mediada también por la conflictividad, dadas las relaciones antagónicas inherentes a toda sociedad de clases, y que configuran un determinado proceso y modo de acumulación.

3. En relación al concepto de neoextractivismo que algunas posiciones esgrimen como noción diferenciadora, vale recordar que desde una perspectiva dialéctica referir un momento en base al prefijo "neo" es por sí mismo obvio y evidente, por cuanto la dialéctica implica precisamente una dinámica cambiante. Por lo tanto, lo "neo" resultaría redundante, debido a que cada nuevo momento del proceso dialéctico implicaría un "neo"-momento. Solo desde miradas que fijan la realidad y la conciben más bien estática, asume el prefijo "neo" algún sentido por cuanto con él se refieren al cambio como una novedad. Claramente no es el caso si entendemos al proceso extractivo como un componente esencial del proceso de acumulación moderno, en donde el cambio y la novedad son unas de sus definiciones fundantes. 
Pero en las últimas décadas, la discursividad sobre la problemática ambiental y territorial se ha visto inundada por el concepto "extractivismo". Ha entrado en escena superando las disquisiciones previas que remitían la problemática ambiental fundamentalmente a desajustes de planificación, organización o hasta de conductas individuales para poner en el centro de la escena al modelo de desarrollo y sus injusticias geopolíticas, o por lo menos a ciertos aspectos de dicho modelo como la fuente primordial a partir de donde poder entender la "crisis ambiental" del presente. Sin embargo, previo al concepto de extractivismo, los pensamientos más lúcidos y críticos ya habían puesto sobre el tapete la centralidad del desarrollo, pero fueron voces escasas y casi solitarias.

Varios/as autores/as muy citados/as apelan hoy al concepto de extractivismo como explicación de la sociedad latinoamericana actual.

A comienzos del siglo XXI, pasados quinientos años, la intensidad renovada por la apropiación y explotación de los recursos minerales se expresa en la multiplicación de mega proyectos de extracción en todo el continente. Pero, a diferencia de épocas pasadas, las condiciones tecnológicas permiten ahora prescindir de grandes contingentes de fuerza de trabajo. Las poblaciones locales, entonces, se transforman en víctimas de procesos de vaciamiento territorial que las excluyen de sus lugares de pertenencia, al mismo tiempo en que destruyen a los ecosistemas con los cuales han convivido, a veces, desde tiempo inmemorial. (...) En el siglo XXI latinoamericano, la disputa por el control de los recursos naturales es un tema álgido de derechos humanos (Alimonda, 2011: 12).

Al extractivismo debemos tanto la pobreza como las crisis económicas, así como el modelo democrático y de convivencia.

La historia de la región nos cuenta que este proceso extractivista ha conducido a una generalización de la pobreza, ha dado paso a crisis económicas recurrentes, al tiempo que ha consolidado mentalidades "rentistas". Todo esto profundiza la débil y escasa institucionalidad democrática, alienta la corrupción, desestructura sociedades y comunidades locales, y deteriora gravemente el medio ambiente. (...)

Lo cierto es que la gran disponibilidad de recursos naturales que caracteriza las economías primario-exportadoras, particularmente si se trata de minerales o petróleo, tiende a distorsionar la estructura económica y la asignación de los factores productivos; redistribuye regresivamente el ingreso y concentra la riqueza en pocas manos. Esta situación se agudiza por una serie de procesos endógenos de carácter "patológico" que acompañan a la abundancia de estos recursos naturales (Acosta, 2011: 83).

En función de esto, y desde una lectura alternativa al predominante discurso "extractivista" nos preguntamos cuál/es es/son el/los concepto/s más pertinentes para dar un debate dialéctico sobre el proceso de despojo, entendiéndolo como un análisis central de la confrontación con el pensamiento científico hegemónico orientado a profundizar la instrumentalización de la naturaleza a escalas cada vez más profundas. A su vez, en esta tarea indagamos qué tipo de importancia tiene, en el contexto actual, recuperar las nociones de capitalismo y lucha de clases y qué nociones o perspectivas podemos incorporar para complejizar este tipo de reflexiones.

\section{II. ¿EL EXTRACTIVISMO COMO FENÓMENO AUTÓNOMO O EL PROCESO EXTRACTIVO COMO MOMENTO DIALÉCTICO DEL PROCESO DE ACUMULACIÓN?}

El carácter autónomo del extractivismo, en el sentido que desde esta práctica puede explicarse tanto el patrón de desarrollo como el derrotero político y hasta la división internacional de trabajo, es ampliamente compartido por la bibliografía actual sobre el tema. Pero además aflora en muchos escritos su carácter novedoso, como nueva forma de producción que si bien puede guardar ciertos lazos con el pasado, asume todas sus características y fuerzas en este presente extractivista.

En términos geopolíticos, la opción extractivista que hoy busca implantarse en la región latinoamericana, desde México a la Argentina, responde a una nueva división territorial y global del trabajo, basado en la apropiación irresponsable de los recursos naturales no renovables, que ha dado lugar 
a nuevas asimetrías económicas, políticas y ambientales entre el Norte y el Sur. En este sentido, el extractivismo resultante no es un destino, es una opción políticaytambién civilizatoria, asumidapor los diferentes gobiernos, que va reconfigurando negativamente nuestros territorios y economías y genera una nueva dependencia: cada vez exportamos más materias primas, lo cual aparece reflejado en la concentración económica, en la especialización productiva, así como en la consolidación de enclaves de exportación, rasgos que históricamente fueron criticados tanto por el desarrollismo como por el marxismo. Esta demanda de materias primas o de bienes de consumo hacia los países dependientes ha conducido a un vertiginoso proceso de reprimarización de la economía latinoamericana que afecta no solo a países como Bolivia, Ecuador o Perú, que cuentan con una fuerte tradición extractivista, sino incluso a aquellos países con patrones económicos más diversificados, como Brasil y Argentina (Svampa, 2011: 186).

La subordinación en el presente a la globalización es letra distintiva, a pesar que existe una más que abundante bibliografía que define a la mundialización (o globalización) como inherente a la misma modernidad. Al mismo tiempo, la apropiación en grandes volúmenes de recursos naturales es por otro lado el signo que definiría al extractivismo. Sin embargo, podríamos considerar, por el contrario, que el incremento en la cantidad extraida está fundamentalmente definida por la acumulación histórica de un patrón tecnológico creciente que nace con el ethos moderno de "el hombre amo y señor de la naturaleza", principio que ya el propio Francis Bacon (1561-1626) en "Novum Organum" declaraba como marca distintiva y disruptiva de la naciente sociedad moderna, al romper sus lazos con la determinación medieval.

En su sentido estricto, los extractivismos son la apropiación de grandes volúmenes de recursos naturales, o bajo procedimientos muy intensivos, donde la mitad o más son destinados a la exportación a los mercados globales. Se refiere a casos como la megaminería a cielo abierto, las plataformas petroleras en la Amazonia, o los monocultivos de soja. Digo en sentido estricto porque este uso del término responde a su historia, a los usos de los movimientos sociales que reaccionaban contra esos emprendimientos por sus impactos, y al dejar en claro que implican una subordinación a la globalización (Gudynas, 2016). El carácter autónomo del extractivismo, junto a su novedad, se colige muy ajustadamente con una de las más ilustrativas definiciones de las últimas décadas como es aquella referida a la "sociedad del riesgo". Este riesgo aparecería solo en esta post-modernidad como diferencia de las "seguras" sociedades hijas de la industrialización que nacieron al calor de las revoluciones y se consolidaron con las guerras mundiales, confrontaciones políticas de diversa índole y más revoluciones.

Así, quince años antes de la finalización del siglo, se declaraba la emergencia y constitución de la "sociedad del riesgo", refiriendo con ello a "una civilización que se pone en peligro a sí misma" (Beck, 1985: 16). El planteo de Beck apuntó a resaltar una serie de cambios decisivos que la problemática ecológica de la Modernidad acusaba en el último tercio del siglo XX. Por entonces, se hace notorio un salto crucial en la escala de los "impactos ambientales". Queda definitivamente atrás la época en la que los daños y consecuencias no deseados y/o no previstos de la acción social sobre el ambiente tenían un efecto temporaly geográficamente circunscripto; se abre paso una nueva era signada por la globalización de los mismos. (...)

Lejos de constituir un freno, los cada vez más amenazadores 'impactos' derivados de la gestión capitalista de la Naturaleza, se convierten en un nuevo y dinámico ámbito para la valorización y expansión del capital. La reciente consagración de la "economía verde" en la Cumbre de Río+20 constituye el epígono de esa trayectoria (Machado Aráoz, 2013: 120).

En síntesis, el extractivismo, bajo un manto de novedad, se presenta como un modelo autónomo distinguido de otras variables que hasta el momento han sido utilizadas para explicar el sistema dominante. A su vez, al vincular el extractivismo con la globalización y la ruptura con lo local, eclipsa las antiguas teorizaciones sobre la división internacional del trabajo.

En función de las reflexiones precedentes, y teniendo en cuenta la importancia del rol que ocupa la naturaleza y lo territorial en la constitución de la modernidad capitalista, podríamos dejar de hablar entonces (desde una perspectiva alternativa) del extractivismo como fenómeno "autónomo" para comenzar a considerar al "proceso extractivo" en tanto componente característico del régimen de acumulación contemporáneo, en el cual los recursos pasan a ser antes que nada factores de producción para la maximización de las ganancias. Es entonces en 
la modernidad capitalista, cuando el "infinito invade este mundo", que el ansia y la capacidad de extracción de la naturaleza y transformación del territorio se maximiza y crece exponencialmente al amparo de la "racionalidad instrumental". Este proceso extractivo que responde al principio ideológico-cultural de maximización de las ganancias variará en su expresión y modo de articulación a medida que los procesos de producción económica y reproducción social y política vayan evolucionando (Marx, [1857] 1978, [1867] 1998), de manera que lo que ayer no era extraíble o transformable, hoy sí ya pueda serlo; y de lo que ayer no era una necesidad, hoy se erija como tal.

Es decir que, más que hablar del extractivismo como fenómeno "neo" en tanto emergente original del presente, quizás sería más preciso enunciar al proceso extractivo en tanto componente fundamental del proceso histórico de acumulación. Esto implica estar aludiendo a una construcción procesual en la cual ni todo es absolutamente nuevo, ni tampoco todo es siempre igual. De esta manera es como pueden analizarse los procesos de renovación y ruptura pero sin dejar de ver la continuidad profunda de ciertos principios y variables que define a toda la modernidad capitalista.

Asimismo, y refiriéndonos ya al proceso sociohistórico regional es que podemos afirmar, más bien confirmar, que la historia del desarrollo de los países latinoamericanos ha sido definida primariamente por la ecuación capital-recursos naturales/territorio, por cuanto emergieron al mundo moderno con un papel predominante de dadores de materias primas, ya sea recursos minerales o agropecuarios. El propio proceso inicial de ocupación europea del continente americano así lo marca claramente, a pesar de ciertos olvidos que pareciera caracterizar la reflexividad contemporánea respecto a los llamados bienes comunes.

Los escritos de Cristóbal Colón en su “Diario de Viajes" (1492), no deja lugar a dudas. Vale aquí trascribir una de sus claras y contundentes afirmaciones:

"Yo estaba atento y trabajaba de saber si había oro, y vide que algunos dellos traían un pedazuelo colgando en un agujero que tenían a la nariz, y por señas pude entender que yendo al Sur o volviendo la isla por el Sur, que estaba allí un Rey que tenía grandes vasos dello, y tenía muy mucho" (porque) "del oro se hace tesoro, y con él quien lo tiene hace cuanto quiere en el mundo y llega a que echa las ánimas al Paraíso".
Es claro que el mismísimo "descubrimiento" y colonización llevaba en su impronta el objetivo de aprovecharse de los recursos materiales y humanos disponibles en las tierras más allá del Viejo Mundo para que sirvieran de incentivo y estímulo al proceso de acumulación capitalista de la Europa moderna naciente.

El hoy llamado extractivismo, es, como se dijo, en realidad intrínseco, cuanto menos ${ }^{4}$, a la modernidad misma y muy especialmente al "nacimiento" de Latinoamérica y el resto de la periferia como resultado de la expansión europea moderna. Es sin dudas en la modernidad cuando el usufructo de la naturaleza y el espacio se intensifican exponencialmente para ponerlo al servicio de la ganancia. En todo caso lo que ha variado en estas últimas décadas, además de las herramientas tecnológicas, fue una presencia complementaria en mayor o menor medida de algún proceso parcial de industrialización. De ahí que aquellos que la definen como extractivista (o neoextractivista) están de alguna manera soslayando la historia latinoamericana y de la propia modernidad, planteando como novedad un proceso que define a toda la trayectoria de"acumulación dependiente" del subcontinente americano.

La territorialización capitalista es, por propia definición, sinónimo de instrumentalización diferencial del territorio que conlleva en sí mismo los mecanismos extractivistas. Extractivismo entonces, debería significar no solo la extracción de componentes de la naturaleza para el sostenimiento, sino un momento asociado con el proceso de acumulación, ganancia y desigualdad de clases. El proceso extractivo de la modernidad capitalista es consecuencia precisamente de la racionalidad instrumental que se constituye con la propia modernidad y no sólo en las últimas décadas neoliberales. Instrumentalidad, por cuanto la naturaleza pasa a ser primordialmente un objeto de usufructo en tanto instrumento esencial para la construcción del "confort" (ideario de felicidad según la razón subjetiva) (Adorno y Horkheimer, [1947] 1971; Galafassi, 2004). Este proceso extractivo que responde a la maximización de las ganancias variará en su expresión y modo de articulación a medida que los procesos de producción económica y reproducción social y política vayan evolucionando, de manera que lo que ayer no era extraíble o transformable, hoy sí ya pueda serlo; $y$ de lo que ayer no era una necesidad, hoy se erija como tal.

La particular conjunción entre tecnología y territorio constituye un eje clave de la competencia internacional a la vez que pilar fundamental en el

4. Decimos "cuanto menos" dado que, y como afirmamos más arriba, el ser humano como especie se constituye cultural e históricamente a partir de su capacidad diferencial para la utilización de la naturaleza a través del proceso extracción-producciónconsumo. 
proceso de construcción de hegemonía. Las disputas internas al capital, disputas por el grado de participación en la distribución de los beneficios, se expresan cada vez más fuertemente, tanto en el desarrollo tecnológico como en la carrera por la búsqueda y transformación de territorios, ya sea para la extracción de los recursos-insumos como para la construcción de mercados de consumo (de esos recursos extraídos y transformados). Esto viene generando relaciones desiguales entre los territorios y las naciones gestando situaciones diferenciales de desarrollo, subdesarrollo, dependencia, desigualdad y subordinación.

Si la propia acumulación originaria se basó en la apropiación por la fuerza (mediación violenta) de tierras y recursos para convertirlos en la matriz esencial de arranque del sistema capitalista de producción, su evolución posterior no estuvo tampoco ajena a esta ecuación expropiatoria (lo que hoy se denomina "extractivismo"). Esta acumulación, basada en la predación y la violencia sin disimulo, en un sector (clase social y territorio), mediada por la desposesión de otro adquiere entonces en la actualidad una evidente visibilidad, cuando el agotamiento de muchos recursos está llamando la atención incluso al propio capital. ${ }^{5}$

Fue muy intensa la discusión sobre estos tópicos en las décadas previas a la instalación del neoliberalismo y nos remiten de alguna manera a las discusiones actuales sobre el desarrollo y el extractivismo. Las discusiones y reflexiones sobre la relación metrópoli-satélite, desarrollo-subdesarrollo, liberación-dependencia, civilización-formación social, etc., se centraban justamente en la discusión sobre la producción y distribución de los recursos, que incluye obviamente todo lo referido a la actividad extractiva, pues no hay producción sin extracción. ${ }^{6}$ Si el hoy llamado extractivismo no estaba presente como concepto tiene que ver, por un lado, con la todavía escasa sensibilidad ambiental de aquellos años, pero también, por otro lado, con la secuencia intelectual obvia que remite a la renovación permanente de las categorías y de su capacidad de interpelación de la realidad en dialéctica relación con los procesos sociopolíticos.

Sin embargo, esta renovación permanente no implica desconocer o no reconocer la existencia de un modo de acumulación particular. Por el contrario, lo ideal sería recuperar conocimientos y discusiones previas sobre su caracterización y profundizar en su análisis. Un ejemplo interesante es el aporte de Federici ([2004] 2010) sobre el rol de las mujeres como reproductoras de la fuerza de trabajo y sobre la necesidad del aniquilamiento de miles de mujeres consideradas "brujas", en el proceso constitutivo del capitalismo como sistema dominante. Las mujeres al ser consideradas como máquinas de producción de nuevos trabajadores también son parte del proceso dialéctico acumulación-producción-extracción. Así, la innovación intelectual, dentro de los estudios dialécticos y complejos estructurados sobre la reflexión del modo de acumulación, es una tarea fundamental para continuar re-pensando en profundidad el mundo en el que vivimos.

En resumen, el extractivismo implica una mirada sobre lo emergente, cuando lo importante sigue siendo una perspectiva de raíz, de la fuente misma de los sucesos. Entonces, la recuperación del análisis del capitalismo como modo de acumulación y de la lucha de clases como conflicto social inherente, forma parte tanto de la disputa política como de la disputa epistemológica, en el contexto actual. Para estas disputas, no es suficiente elegir un tema de investigación/estudio/enseñanza de relevancia histórico-coyuntural; sino que también es clave el modo de analizar ese tema y los presupuestos que cada herramienta conlleva.

\section{ACUMULACIÓN- PRODUCCIÓN-EXTRACCIÓN COMO PROCESO DIALÉCTICO}

Acumulación primitiva, reproducción ampliada y nuevos cercamientos representan una ecuación importante a la hora de entender la estrategia extractiva y de apropiación de la naturaleza y construcción del territorio en la sociedad capitalista. En esta ecuación la conceptualización de bienes comunes se opone claramente al proceso de privatización de la existencia y la consecuente transformación en mercancías, tanto de los objetos producto del trabajo como del trabajo

\footnotetext{
5. Vale aclarar que este proceso de crecimiento y desarrollo basado en la desposesión, el saqueo y el pillaje no es privativo del capitalismo, aunque el ritmo y la eficiencia del actual proceso de predación es inhallable en cualquier ejemplo del pasado. De diversas formas y expresiones, se lo registra en reiteradas oportunidades en la historia de occidente. Vale citar solo algunos ejemplos, la conquista sucesiva de círculos concéntricos como nuevas zonas de pillaje en el período de la decadencia romana (cfr. Chaunu, Piere. (1991). Historia y decadencia, Madrid, Granica); o la llamada "revolución industrial en la baja edad media", asentada, entre otras cosas, en otro proceso de pillaje colonial motorizado por las Cruzadas (cfr, Gimpel, Jean. (1982). La revolución industrial en la Edad Media. Madrid, Taurus; Gaudin, Thierry. (1988). Les metamorphoses du futur. Paris, Económica); o las llamadas crisis de subproducción que terminan agotando los recursos naturales, características de economías con alta predominancia del sector agrícola.
}

6. Para esto revisar por ejemplo: Furtado, 1964; Gunder Frank, 1970; Ribeiro, 1972; Marini, 1973; Bambirra, 1974 y Dos Santos, 1978. 
mismo. Los nuevos cercamientos entonces implican la apropiación de aquellas porciones de territorio y espacios de vida aún no incorporados plenamente a la lógica del capital, reeditando así algunos de los procesos de la llamada acumulación primitiva que conviven de esta manera con los mecanismos predominantes de la reproducción ampliada (Galafassi, 2014 y 2015).

Es así entonces que debemos además considerar lo que se ha llamado la "segunda contradicción del capital" (O'Connor, 1988, 2001), como aquel proceso que trata en tanto mercancía a la naturaleza y el espacio, de tal manera de poder incluirlos en su ecuación instrumental. La tendencia es al socavamiento de la propia base natural de sustentación del sistema productivo, dado que el capital no puede prever los costos de reproducción de la naturaleza en pos de una sustentabilidad real, debido a que afectaría claramente la tasa de ganancia. En este esquema de racionalidad instrumental, segunda contradicción y conjunción de procesos de acumulación, es que se vienen definiendo históricamente toda una serie diversa de recursos estratégicos que se relacionan dialécticamente, por cuanto, por un lado, son aquellos que la dinámica global del capital define como recurso demandado en un momento histórico determinado; $y$, por otro, como aquellos que las condiciones ecológicas regionales determinan como aptos para ser producidos o extraídos en cada territorio.

Podemos hablar entonces de un proceso extractivo que se va transformando históricamente en base a la innovación tecnológica permanente y a la propia dinámica de cambio del proceso de acumulación. En esta continuidad extractiva en función del proceso de acumulación, el caucho, es un ejemplo histórico en la América Tropical, la plata lo es en la América Andina, el quebracho en América Subtropical. Más contemporáneo, la explotación de los hidrocarburos y de minerales no deja de generar conflictos socio-políticos y territoriales, donde entran en juego intereses geoestratégicos estadounidenses, capitales multinacionales de base europea y gobiernos con orientación popular-reformista o conservadora. ${ }^{7}$ Sin ir más lejos, es importante no dejar pasar los importantes conflictos geopolíticos derivados por la posesión de los yacimientos de gas y petróleo en las recientes historias de Venezuela y Bolivia, ${ }^{8}$ más la llamada Guerra del Agua, también en Bolivia, ${ }^{9}$ o las más recientes disputas en torno a la potencial energía hidroeléctrica de los ríos patagónicos tanto como los cuestionamientos al avance de la frontera hidrocarburífera con el fracking en dicha región, ${ }^{10}$ los cuales muestran de forma elocuente lo central de esta cuestión. Primordial es también mencionar el proceso creciente de sojización de América del Sur, que arrasó con ecosistemas, agrosistemas y culturas, constituyéndose no sólo en la extracción de un recurso en base a su "oportunidad" en términos de su demanda por las naciones más industrializadas (alimento de ganado y biodiésel) sino que también en la aplicación de la tecnología más concentrada y asociada a fuertes niveles de dependencia.

Alienación socio-ecológica, "extractivismo" histórico e instrumentalización de la razón están en la base y las consecuencias de todos estos procesos de acumulación basados en la territorialización extractiva desde que el continente americano es "descubierto" por el capital europeo. Es así, que la caracterización que hiciera Galeano ([1971] 2015: 16) en Las Venas Abiertas de América Latina en la década de 1970 sigue absolutamente vigente, poniendo en entredicho los supuestos "descubrimientos intelectuales" del extractivismo o neo-extractivismo tan en boga actualmente.

Es América Latina, la región de las venas abiertas. Desde el descubrimiento hasta nuestros días, todo se ha trasmutado siempre en capital europeo o, más tarde, norteamericano, y como tal se ha acumulado y se acumula en los lejanos centros de poder. Todo: la tierra, sus frutos y sus

7. Es importante aquí refrescar algunos datos. El $25 \%$ del crudo comercializado a nivel internacional en 2005 era comprado por EEUU, quien solo representaba el 9\% de la producción mundial de petróleo. La Unión Europea importa el $80 \%$ del petróleo que consume y Japón compra al exterior casi el 100\%. Entre las tres potencias producen solo el $12 \%$ del total a nivel mundial, aunque en su consumo se va el 50\% del producido a nivel mundial e importan el $62 \%$ del comercio internacional (Beinstein, 2004). Más concretamente, vale lo dicho por el ex presidente de los EEUU: "...America is now more dependent on foreign oil than a time in its history. In 1973, the country imported 36 percent of its oil needs. Today, the U.S. imports 56 percent of its crude oil (...) The U.S. bill for foreign oil has more than doubled from last year..." (Bush, G. W. (2000) On The Issues Energy, 4president.org, http:// www.4president.org/issues/bush2000/bush2000energy.htm). Pese a que en las últimas décadas, con la técnica de la fractura hidráulica y la extracción de hidrocarburos denominados no convencionales (shale oil/gas o tight gas, entre los más conocidos), EEUU ha incrementado la extracción de crudo (EIA: Hydraulic fracturing accounts for about half of current U.S. crude oil production, https://www.eia.gov/todayinenergy/detail.php?id=25372\#), la lógica se mantiene intacta. En este caso, lo que están distribuyendo a nivel mundial es el conocimiento técnico y el paquete tecnológico del fracking, que es experimental y se encuentra protegido por el secreto comercial en EEUU, por lo cual poco se puede saber de las consecuencias territoriales de su aplicación en cualquier parte del mundo (Pérez Roig, 2014).

8. cfr. Villegas Quiroga (2003); Escobar de Pavón, Silvia (2004); Lander (2004).

9. Kruse (2005).

10. cfr. Di Risio, Diego et. Al (2012); Pérez Roig (2012); Riffo (2016). 
profundidades ricas en minerales, los hombres ysu capacidad de trabajo y de consumo, los recursos naturales y los recursos humanos. El modo de producción y la estructura de clases de cada lugar han sido sucesivamente determinados, desde fuera, por su incorporación al engranaje universal del capitalismo. A cada cual se le ha asignado una función, siempre en beneficio del desarrollo de la metrópoli extranjera de turno, y se ha hecho infinita la cadena de las dependencias sucesivas, que tiene mucho más de dos eslabones, y que por cierto también comprende, dentro de América Latina, la opresión de los países pequeños por sus vecinos mayores $y$, fronteras adentro de cada país, la explotación que las grandes ciudades y los puertos ejercen sobre sus fuentes internas de víveres y mano de obra (Hace cuatro siglos, ya habian nacido dieciséis de las veinte ciudades latinoamericanas más pobladas de la actualidad).

Para finalizar, en definitiva, la clave diferenciadora no está en pensar en extractivismo sino en acumulación, entendiéndolo como la articulación entre el despojo, o sea los mecanismos de la acumulación primitiva, y la reproducción ampliada del capital. El extractivismo es solo un instrumento para la acumulación capitalista. Por lo tanto debe ser tratado conceptualmente como tal. El eje está en la lógica de acumulación y el extractivismo es solo una herramienta para el inicio de este proceso. Para terminar con el extractivismo es necesario discutir todo el proceso complejo y dialéctico de la acumulación y sus diferentes facetas y solo en este entramado discutir la etapa extractiva del capital, por cuanto el proceso extractivista es parte de la totalidad y si bien tiene sus especificidades solo se explica en su sentido integro en función de esa totalidad.

\section{BIBLIOGRAFÍA}

Acosta, A. (2011). Extractivismo y neoextractivismo: dos caras de la misma maldición. En AAVV. Más allá del desarrollo, Grupo Permanente de Trabajo sobre Alternativas al Desarrollo. Quito. Ecuador: Universidad Politécnica Salesiana/Fundación Rosa Luxemburg. pp. 81-118

Adorno, T. y Horkheimer, M. ([1947] 1971). Dialéctica del Iluminismo. Buenos Aires. Ed. Sur.

Alimonda, H. (2011). La naturaleza colonizada. Ecología política y minería en América Latina. Buenos Aires. CLACSO-Ciccus.

Bambirra, V. (1974). El capitalismo dependiente latinoamericano. México: Siglo XXI.

Beinstein, J. (2004). Estados Unidos en el centro de la crisis mundial, en, Enfoques Alternativos, n²7, Buenos Aires, noviembre de 2004.

Bacon, F. (1620). Novum Organum (varias ediciones).

Chaunu, P. (1991). Historia y decadencia. Madrid. Granica.

Colón, Cristóbal (1492). Registro de viaje para el sábado 13 de octubre de 1492. Recuperado de: https:// es.wikisource.org/wiki/Diario_de_a_bordo_del_ primer_viaje_de_Crist\%C3\%B3bal_Col\%C3\%B3n

Di Risio, D., Gavaldà, M., Pérez Roig, D. \& Scandizzo, H. (2012). Zonas de sacrificio. Impactos de la industria hidrocarburífera en Salta y Norpatagonia. Buenos Aires. América Libre.

Dos Santos, T. (1978). Imperialismo y dependencia. México. Era.

Escobar de Pavón, Silvia (2004). Ajuste y liberalización, las causas del conflicto social, en OSAL - Observatorio Social de América Latina n 12, pp. 47-56.

Federici, S. ([2004] 2010). El Calibán y la bruja. Mujeres, cuerpo y acumulación originaria. Madrid: Traficantes de sueños.

Furtado, C. (1964). Desarrollo y subdesarrollo. Buenos Aires. Eudeba.

Galafassi, G. (2004). Naturaleza, sociedad, alienación. Ciencia y desarrollo en la modernidad. Montevideo. Ed. Nordan-Comunidad. 
Galafassi, G. (2014). Acumulación, Hegemonía y Conflictos Sociales en la historia regional Patagónica: de la ganadería extensiva a la megaminería. PolHis. Dossier Acciones Colectivas, Resistencias y Movimientos Sociales en América Latina, n 37, pp. 1-19. Disponible en: http://historiapolitica.com/datos/biblioteca/ protestas_galafassi.pdf

Galafassi, G. (2015) Acumulación, conflictos sociales y políticos de Estado en América Latina en las últimas décadas. Cambios y rupturas en el escenario regional. Revista Encuentros Latinoamericanos, UDELARMontevideo. Disponible en: http://enclat.fhuce.edu.uy/

Galeano, E. ([1971] 2015). Las venas abiertas de América Latina. Buenos Aires: Siglo Veintiuno Editores.

Gaudin, T. (1988). Les metamorphoses du futur. Paris. Económica.

Gimpel, J. (1982). La revolución industrial en la Edad Media. Madrid. Taurus.

Gudynas, E. (2016). Las consecuencias del extractivismo en América Latina. Entrevista en La izquierda diario, 13 de mayo de 2016.

Gunder Frank, A. (1970). Capitalismo y Subdesarrollo en América Latina. Buenos Aires. Signos.

Kruse, T. (2005). La guerra del agua en Cochabamba, Bolivia: terrenos complejos, convergencias nuevas; en, Enrique de la Garza Toledo (comp.), Sindicatos y nuevos movimientos sociales en América Latina. Buenos Aires. CLACSO.

Lander, E. (2004). Venezuela: proceso de cambio, referéndum revocatorio y amenazas territoriales, en OSAL - Observatorio Social de América Latina, no 13, pp. 57-66.

Machado Aráoz, H. (2013). Crisis ecológica, conflictos socioambientales y orden neocolonial: Las paradojas de NuestrAmérica en las fronteras del extractivismo. Rebela, Revista Brasilera de estudos Latino-Americanos, vol. 3, no 1, pp. 118-155.

Marini, R. M. (1973). Dialéctica de la dependencia. México. Era.

Marx, K. ([1844] 1968). Manuscritos economía y filosofía. Madrid. Alianza Editorial.
Marx, K. ([1857] 1971). Formaciones económicas precapitalistas. México. Siglo XXI.

Marx, K. ([1857] 1978). Elementos fundamentales para la crítica de la economía política (Grundrisse) [1857-1858]. México. Siglo XXI.

Marx, K. ([1867] 1998). El capital, tomo 1. México: Siglo XXI.

O'Connor, J. (1988). Capitalism, nature, socialism: a theoretical introduction. Capitalism, Nature, Socialism, no 1.

O'Connor, J. (2001). Causas naturales. Ensayos de marxismo ecológico. México. Siglo XXI.

Pérez Roig, Diego (2014). Fracturando Argentina. Promoción y resistencias al avance de los'hidrocarburos no convencionales. En Composto, Claudia y Navarro, Mina Lorena: Territorios en disputa. Despojo capitalista, luchas en defensa de los bienes comunes naturales y alternativas emancipatorias para América Latina. México, D. F.: Bajo Tierra Ediciones. pp. 149-167.

Pérez Roig, Diego (2012). Los hidrocarburos no convencionales en el escenario energético argentino, en Revista Theomai. Estudios críticos sobre el Desarrollo, n²5, pp. 113-127. http://revista-theomai.unq.edu.ar/ NUMERO\%2025/12PerezRoig.pdf

Ribeiro, Darcy. (1972). Las Américas y la civilización: proceso de formación y causas del desarrollo desigual de los pueblos americanos. Buenos Aires. Centro Editor de América Latina.

Riffo, Lorena (2016). Hidrocarburos, fracking y resistencias sociales. Un análisis social de las políticas hidrocarburíferas contemporáneas en Argentina desde la provincia de Neuquén, en Actuel Marx Intervenciones n²0, pp. 71-94.

Svampa, M. (2011). Extractivismo neodesarrollista y movimientos sociales. ¿Un giro ecoterritorial hacia nuevas alternativas? En AAVV. Más allá del desarrollo, Grupo Permanente de Trabajo sobre Alternativas al Desarrollo. Quito. Universidad Politécnica Salesiana/ Fundación Rosa Luxemburg. pp. 185-218.

Villegas C. (2003). Rebelión popular y los derechos de propiedad de los hidrocarburos, en OSAL - Observatorio Social de América Latina n¹2, pp. 27-34. 